\title{
A FRET Assay for Celiac Disease
}

\author{
Sae A Lee, Chul Min Cho, Il Ho Jang and Jung Sook Kang ${ }^{\dagger}$ \\ Department of Oral Biochemistry and Molecular Biology, School of Dentistry, \\ Pusan National University, Yangsan 50612, Korea
}

To provide a basis for a homogeneous fluorescence resonance energy transfer (FRET) immunoassay for celiac disease, we carried out a FRET experiment using guinea pig tissue transglutaminase (tTG) and antibodies to tTG (anti-tTG) purified from rat serum. Fluorescein was utilized as the probe, and a nonfluorescent dye, QSY 7 served as the quencher. We labeled anti-tTG and tTG with fluorescein isothiocyanate and QSY 7 succinimidyl ester, respectively. Fluoresceinlabeled anti-tTG was the donor, and QSY 7-labeled tTG was the acceptor of the FRET experiment. When we titrated fluorescein-labeled anti-tTG with QSY 7-labeled tTG, we observed a large decrease in the steady-state fluorescence intensity, which was due to strong FRET from fluorescein-labeled anti-tTG to QSY 7-labeled tTG. Using time-resolved fluorescence spectroscopy, we could also observe a decrease in the fluorescence lifetime, which confirms the steady-state data. We expect that these results might be useful in the development of a novel fluorescence immunoassay for an easy screening and follow-up of celiac patients.

Key Words: Fluorescence resonance energy transfer, Tissue transglutaminase, Celiac disease, Anti-tTG antibodies

\section{INTRODUCTION}

Celiac disease (CD) is a chronic autoimmune disease of the small intestine triggered by the ingestion of wheat gluten and related protein complexes found in barley and rye. This represents the most frequent food intolerance with some reports estimating a prevalence of up to $1 \%$ worldwide (Catassi and Fasano, 2008). The pathology is characterized by villous atrophy, crypt hyperplasia, and inflammation of the small intestinal mucosa. CD patients show a broad spectrum of clinical manifestations, such as chronic diarrhea, fatigue, weight loss, abdominal distention, malabsorption syndromes including anemia and osteoporosis, infertility, as well as a substantially enhanced risk for the development of a fatal small intestinal lymphoma (Green and Jabri, 2003). Early diagnosis and follow-up of CD are crucial because a lifelong gluten-free diet is the only effective current treatment of $\mathrm{CD}$.

A $\mathrm{T}$ cell-mediated immune response against gluten fractions (gliadins and glutenins) that occurs in individuals bearing the human leucocyte antigen alleles DQ2/8 is considered as the pivotal event in the pathogenesis of CD (Sollid, 2000; Sollid and Jabri, 2013). In addition to the cellular response, CD patients show autoantibodies produced against reticulin, gliadin, endomysium and tissue transglutaminase (tTG), which is the basis for the serological screening of CD (Dieterich et al., 1997; Basso et al., 2009; Chorzelski et al., 1984). Antireticulin antibodies are specific but not sensitive; antigliadin immunoglobulin (Ig) G or IgA

* Received: October 28, 2016 / Revised: December 15, 2016 / Accepted: December 16, 2016

${ }^{\dagger}$ Corresponding author: Jung Sook Kang. Department of Oral Biochemistry and Molecular Biology, School of Dentistry, Pusan National University, Yangsan 50612, Korea.

Tel: +82-51-510-8226, Fax: +82-51-510-8228, e-mail: jsokang@pusan.ac.kr

(C) The Korean Society for Biomedical Laboratory Sciences. All rights reserved.

(C) This is an Open Access article distributed under the terms of the Creative Commons Attribution Non-Commercial License (http://creativecommons.org/licenses/by-nc/3.0/) which permits unrestricted non-commercial use, distribution, and reproduction in any medium, provided the original work is properly cited. 
antibodies have limitations both in sensitivity and specificity (Hill, 2005). Due to the high specificity, antiendomysial antibodies (EMAs) of the IgA class are still regarded as the reference standard for CD-specific antibody detection (Husby et al., 2012). As this diagnostic approach is based on indirect immunofluorescence, using the esophagus of monkeys or umbilical cord as substrate, it is labor-intensive and operatordependent. After Deitrich et al. (1997) discovered that the antigen recognized by EMA is tTG, several enzyme-linked immunosorbent assays (ELISAs) have been developed to determine the anti-tTG antibodies. The first-generation ELISA kits used guinea pig liver as the tTG source and are based on spectrophotometry. The second-generation ELISAs use human recombinant or human purified tTG and are based on indirect chemiluminescence (Basso et al., 2006). At present measurement of IgA antibodies to recombinant tTG is considered the most efficient single serologic test for CD diagnosis (AGA Institute, 2006). In addition, to demonstrate a decrease in IgA anti-tTG titer is regarded as an indirect indicator of dietary adherence and recovery (Hill et al., 2005).

However, definite diagnosis of CD still relies on the intestinal biopsy findings. There is no available serological test that can definitively diagnose or exclude CD. Additionally, no serological test is powerful enough to assess compliance to a gluten-free diet and/or the occurrence of dietary transgressions (Leffler et al., 2007). Besides, 2 to $3 \%$ of CD patients have selective IgA deficiency (Collin et al., 1992). These patients often have false negative IgA anti-tTG assay, so IgG is a diagnostic alternative (Rittmeyer and Rhoads, 1996, Lenhardt et al., 2004). Moreover, the sensitivities for the $\mathrm{tTG}$ assays ranged from $72.5 \%$ to $98.6 \%$ and specificities from $60.3 \%$ to $99.2 \%$ depending on the kit (Suh-Lailam et al., 2016), suggesting the need for not only standardization but also improvement of the sensitivities and specificities of anti-tTG immunoassay.

The purpose of this study is to provide a basis for a homogeneous fluorescence resonance energy transfer (FRET) immunoassay for easy screening and follow-up of celiac patients. tTG and IgG anti-tTG antibodies were labeled with a quencher and an extrinsic fluorophore, respectively. The donor was fluorescein-labeled anti-tTG, and the acceptor was nonfluorescent QSY 7-labelled tTG. We characterized the FRET from fluorescein-labeled anti-tTG to QSY 7labelled tTG.

\section{MATERIALS AND METHODS}

\section{Materials}

tTG and protein A antibody purification kit were obtained from Sigma (St. Louis, MO, USA), and Sephadex-G25M columns were from GE Healthcare (Piscataway, NJ, USA). IgG anti-tTG (whole anti-serum, developed in rats) was kindly provided by Dr. Mauro Rossi (Institute of Food Science, CNR, Avellino, Italy). Fluorescein isothiocyanate and QSY 7 succinimidyl ester were purchased from Invitrogen (Carlsbad, CA, USA), and centricon YM-3 concentrators were supplied by Amicon (Beverly, MA, USA). All other chemicals were reagent grade, and water was deionized with a Milli-Q system.

\section{Absorption and steady-state fluorescence measurement}

Centricon YM-3 concentrators were used to concentrate the aqueous solution of tTG and to change the buffer into $0.1 \mathrm{M}$ sodium bicarbonate buffer $(\mathrm{pH}$ 8.3) because tTG was suppled as a powder lyophilized from a Tris buffer. The anti-tTG was purified using protein A antibody purification kit. The purified anti-tTG was also concentrated using Centricon YM-3 concentrators, and the buffer was changed into $0.1 \mathrm{M}$ sodium bicarbonate buffer ( $\mathrm{pH} 9.0$ ). About $2 \sim 5 \mathrm{mM}$ of stock solutions of fluorescein isothiocyanate and QSY 7 succinimidyl ester were prepared in dimethylformamide. The tTG and anti-tTG were labeled with QSY 7 succinimidyl ester and fluorescein isothiocyanate, respectively, by adding 10 -fold molar excess of the dyes to $1 \mathrm{ml}$ of slowly stirred protein solution in $0.1 \mathrm{M}$ sodium bicarbonate buffer followed by one hour incubation at room temperature. The resulting labeled proteins were separated from the free dye by passing the solution through a Sephadex G-25M column, using 50 $\mathrm{mM}$ Tris, $150 \mathrm{mM} \mathrm{NaCl}, 10 \mathrm{mM}$ ethylenediaminetetraacetic acid, $\mathrm{pH}$ 7.4. The dye to protein molar ratios of the conjugates were determined by measuring the absorbance of the protein-dye conjugates at $280 \mathrm{~nm}$ and at the $\lambda_{\max }$ for the dye. For fluorescein-labeled $\alpha$-tTG, the $\lambda_{\max }$ was $494 \mathrm{~nm}$ $\left(\varepsilon_{494 \mathrm{~nm}}=77,000 \mathrm{M}^{-1} \mathrm{~cm}^{-1}\right)$, and the correction factor for 
measuring the protein was 0.30 . The $\lambda_{\max }$ value for QSYlabeled tTG was $560 \mathrm{~nm}\left(\varepsilon_{560 \mathrm{~nm}}=90,000 \mathrm{M}^{-1} \mathrm{~cm}^{-1}\right)$, and the correction factor was assumed to be 0.22 . Ultraviolet-visible absorption spectra were measured with a Cary 50 Bio spectrophotometer (Varian, Palo Alto, CA, USA). Steady-state fluorescence measurements were carried out using a K2 spectrofluorometer (ISS, Inc., Urbana, IL, USA). The excitation wavelength was $490 \mathrm{~nm}$.

The Förster distance $R_{0}$, which is the distance at which FRET is $50 \%$ efficient, is given by

$$
R_{0}=\left(J(\lambda) \kappa^{2} Q_{D} n^{-4}\right)^{1 / 6} \times 9.78 \times 10^{3} \AA
$$

where $J(\lambda)$ is the spectral overlap integral of donor emission and acceptor absorption, $k^{2}$ is the orientation factor for dipole-dipole interaction, $Q_{D}$ is quantum yield of the donor in the absence of acceptor, and $n$ is the refractive index of the medium. The overlap integral $J(\lambda)$ expresses the degree of spectral overlap between the donor emission and the acceptor absorption:

$$
J(\lambda)=\int_{0}^{\infty} F_{D}(\lambda) \varepsilon_{A}(\lambda) \lambda^{4} d \lambda
$$

where $F_{D}(\lambda)$ is the corrected fluorescence intensity of the donor in the wavelength range $\lambda$ to $\lambda+\Delta$, with the total intensity (area under the curve) normalized to unity, and $\varepsilon_{A}(\lambda)$ is the extinction coefficient of the acceptor at $\lambda$, which is typically in the unit of $\mathrm{M}^{-1} \mathrm{~cm}^{-1}$.

The efficiency of FRET $(E)$ is the fraction of photons absorbed by the donor that are transferred to the acceptor and calculated by

$$
E=1-\frac{F_{D A}}{F_{D}}
$$

where $F_{D}$ and $F_{D A}$ are the fluorescence intensities in the absence and presence of the acceptor, respectively.

\section{Frequency-domain intensity decay measurements}

Measurements were performed with a Chronos phase and modulation spectrometer (ISS, Urbna, IL, USA). The excitation source was a green LED provided by ISS. A $490 \pm 5$ $\mathrm{nm}$ and $532 \pm 17.5 \mathrm{~nm}$ interference filters were used for isolating excitation and emission, respectively. Erythrosin B in water ( $\tau=80 \mathrm{ps}$ ) was utilized as a lifetime standard.

The intensity decays were recovered from the frequencydomain data in terms of a multi-exponential model:

$$
I(t)=\sum_{i=1}^{n} \alpha_{i} e^{-t / \tau_{i}}
$$

where the preexponential factors ai are the amplitude of each component, $\Sigma \alpha_{i}=1.0, \tau_{i}$ are the decay times, and $n$ are the numbers of exponential components. These values were determined by nonlinear least squares analysis as described previously (Lakowicz et al., 1984). Mean lifetimes were calculated by:

$$
<\tau>=\frac{\sum_{i} \alpha_{i} \tau_{i}^{2}}{\sum_{i} \alpha_{i} \tau_{i}}=\sum_{i} f_{i} \tau_{i}
$$

where $f_{i}$ is the fractional steady-state contribution of each component to the total emission, and $\Sigma f_{i}$ is normalized to unity. $f_{i}$ is given by

$$
f_{i}=\frac{\alpha_{i} \tau_{i}}{\sum_{j} \alpha_{j} \tau_{j}}
$$

The best fits were obtained by a minimum value for the goodness-of-fit parameters $\chi_{R}^{2}$ :

$$
\chi_{R}^{2}=\frac{1}{v} \sum_{\omega}\left[\left(\frac{\varphi_{\omega}-\varphi_{c \omega}}{\delta \varphi}\right)^{2}+\left(\frac{m_{\omega}-m_{c \omega}}{\delta m}\right)^{2}\right]
$$

where $v$ is the number of degrees of freedom, and $\varphi_{\omega}$ and $m_{\omega}$ are the experimental phase and modulation, respectively. The subscript $c$ is used to indicate calculated values for assumed values of $\alpha_{i}$ and $\tau_{i}$, and $\delta \varphi$ and $\delta m$ are the experimental uncertainties. Here, the values of $\delta \varphi$ and $\delta m$ were 


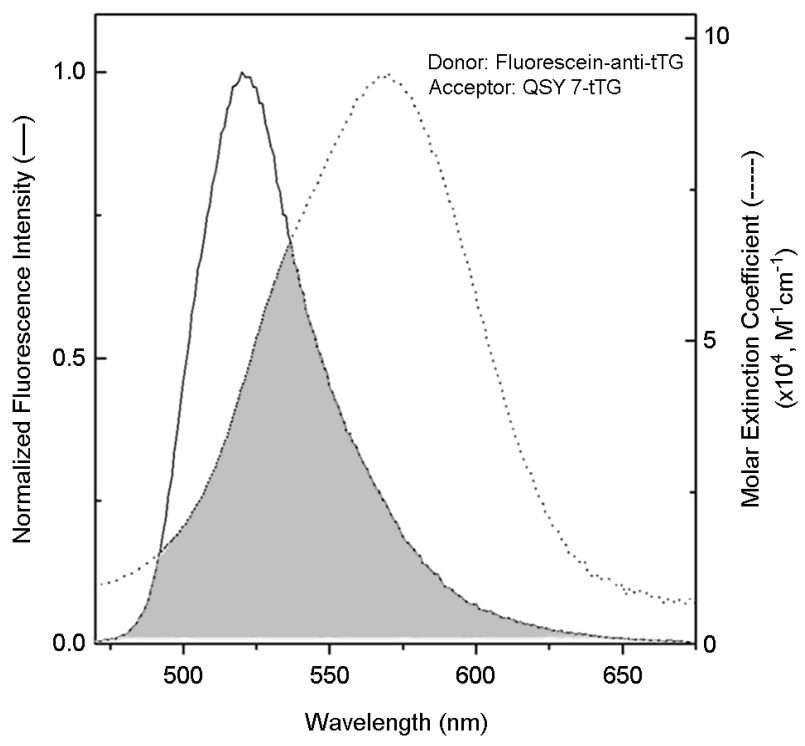

Fig. 1. Emission spectrum (-) of fluorescein conjugated to antitTG and absorption spectrum ( $\cdots)$ of QSY 7 conjugated to tTG.

set at $0.2^{\circ}$ and 0.005 , respectively.

\section{RESULTS}

\section{Steady-state fluorescence spectra}

FRET is transfer of the excited state energy from an initially excited donor to an acceptor. The rate of energy transfer depends upon the extent of spectral overlap of the emission spectrum of the donor with the absorption spectrum of the acceptor, the quantum yield of the donor, the relative orientation of the donor and acceptor transition dipoles, and the distance between the donor and acceptor molecules (Lakowicz, 2006). Because of the simple operation under homogeneous conditions, it has been used as a power tool for investigating a number of molecular interactions, especially protein-protein interactions. Fig. 1 shows the absorption and emission spectra of the fluorescein-antitTG-QSY 7-tTG donor-acceptor pair. As can be seen, there is a significant overlap of the emission spectrum of the donor fluorescein-anti-tTG with the absorption spectrum of the acceptor QSY 7-tTG. The spectral overlap integral $(J(\lambda))$ was used to calculate the Förster distance $R_{0}$ according to Eq. (1), and the result is summarized in Table 1 . The $R_{0}$ value

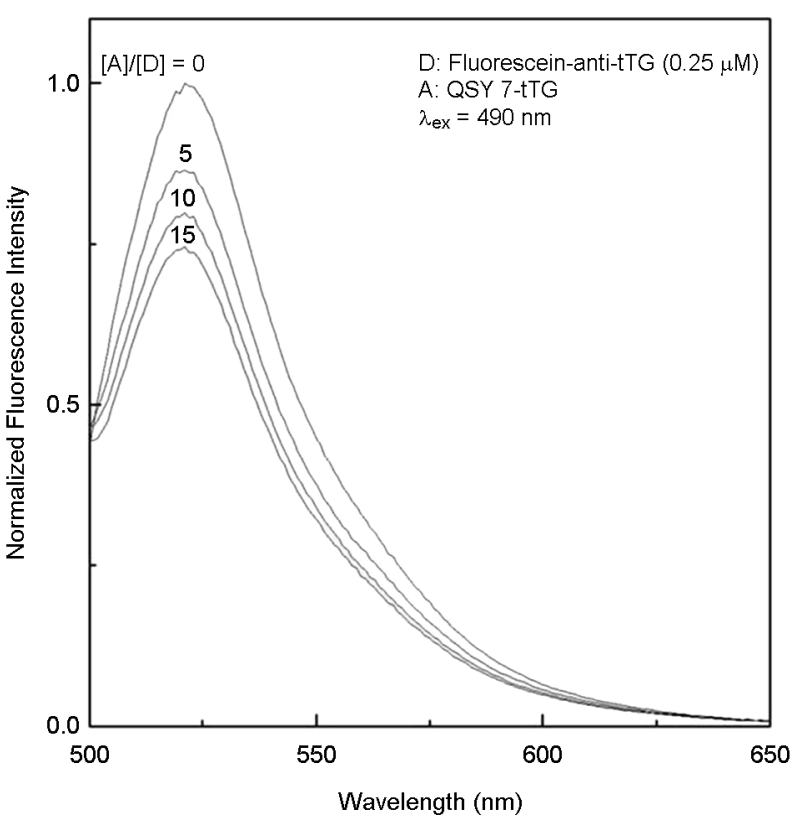

Fig. 2. Emission spectra of the fluorescein-anti-tTG complex in the absence and presence of different concentrations of the QSY 7-tTG complex.

Table 1. Donor quantum yield $\left(Q_{D}\right)$, spectral overlap integral $(J(\lambda))$ and Förster distance $\left(R_{0}\right)$ of fluorescein-anti-tTG-QSY 7-tTG donor-acceptor pair

\begin{tabular}{ccc}
\hline \hline$Q_{D}{ }^{\mathrm{a}}$ & $\begin{array}{c}J(\lambda)^{\mathrm{b}} \\
\left(\times 10^{-13}, \mathrm{M}^{-1} \mathrm{~cm}^{3}\right)\end{array}$ & $\begin{array}{l}R_{0}^{\mathrm{b}} \\
(\AA)\end{array}$ \\
\hline 0.85 & 4.668 & 64.8 \\
\hline
\end{tabular}

${ }^{\mathrm{a}}$ Fluorescein in $0.1 \mathrm{M} \mathrm{NaOH}(Q=0.95)$ was used as a quantum yield reference.

${ }^{\mathrm{b}} J(\lambda)$ and $R_{0}$ were calculated according to Eqs. (2) and (1), respectively.

for the fluorescein-anti-tTG-QSY 7-tTG donor-acceptor pair was $64.8 \AA$. In the calculation of $R_{0}$, the value of $k^{2}$ was $2 / 3$, and that of the refractive index $(n)$ was taken as 1.5 (Lakowicz, 2006). Fig. 2 shows the emission spectra of fluorescein-anti-tTG upon adding the acceptor QSY 7-tTG. The steady-state fluorescence intensities of fluorescein-antitTG with a peak at about $520 \mathrm{~nm}$ decreased progressively with an increase in QSY 7 concentration. Fig. 3 shows the efficiency of FRET $(E)$, which was calculated using Eq. (3) from the peak intensities of fluorescein-anti-tTG at about $520 \mathrm{~nm}$ in Fig. 2. The FRET efficiency $E$ showed evidence 


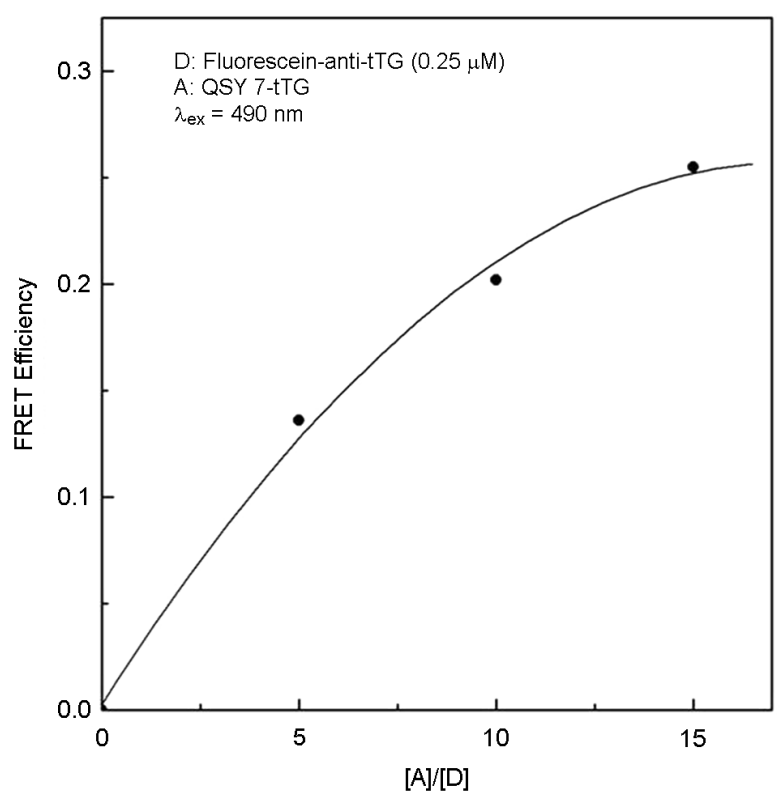

Fig. 3. FRET efficiency $(E)$. Data are from Fig. 2, and the $E$ values were calculated using Eq. (3).

of saturation, with a maximum of about $26 \%$ quenching at a QSY 7-tTG to fluorescein-anti-tTG molar ratio of 15:1.

\section{Intensity decays}

To provide further evidence for FRET from fluoresceinanti-tTG to QSY 7-tTG, we carried out frequency-domain intensity decay measurements. The intensity decays were analyzed in terms of the multiexponential model according to Eqs. (4)-(7), and the results are summarized in Table 2. In the absence of the acceptor, the intensity decay of fluorescein was heterogeneous and showed two exponential decays with a mean decay time of 3.48 ns. Upon FRET the intensity decays became faster (Fig. 4 and Table 2). As the acceptor concentration increased, the frequency responses shifted to high modulation frequencies, which indicate a decrease in the mean decay time. However, the decreases in the lifetimes were not as significant as those in the steadystate intensities. We interpret this that not only dynamic but also static quenching occurred in the fluorescein-anti-tTGQSY 7-tTG donor-acceptor pair.

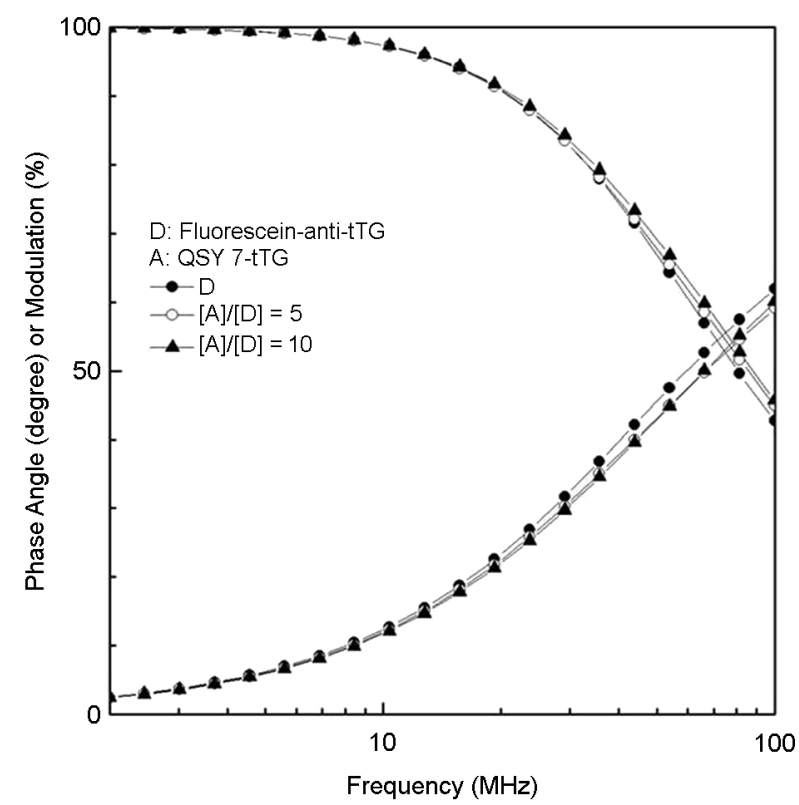

Fig. 4. Frequency-domain intensity decays of the fluorescein-antitTG complex in the absence and presence of different concentrations of the QSY 7-tTG complex.

Table 2. Multiexponential intensity decay analysis

\begin{tabular}{cccccc}
\hline \hline$[\mathrm{A}] /[\mathrm{D}]$ & $\begin{array}{c}\tau_{i} \\
(\mathrm{~ns})\end{array}$ & $\alpha_{i}$ & $f_{i}^{\mathrm{a}}$ & $\begin{array}{c}<\tau^{\mathrm{a}} \\
(\mathrm{ns})\end{array}$ & $\chi_{R}^{2 \mathrm{~b}}$ \\
\hline 0 & 1.591 & 0.148 & 0.068 & 3.48 & 1.23 \\
& 3.806 & 0.852 & 0.932 & & \\
5 & 1.718 & 0.303 & 0.154 & 3.39 & 1.53 \\
& 4.113 & 0.697 & 0.846 & & \\
10 & 0.847 & 0.125 & 0.033 & 3.24 & 1.28 \\
& 3.583 & 0.875 & 0.967 & & \\
\hline
\end{tabular}

${ }^{\mathrm{a}}$ Fractional intensities $f_{i}$ and mean lifetimes $\langle\tau\rangle$ were calculated using Eqs. (6) and (5), respectively.

${ }^{\mathrm{b}}$ The $\chi_{R}^{2}$ values were calculated by the software provided by ISS, Inc.

\section{DISCUSSION}

In this report, we calculated the Förster distance and the efficiency of the FRET from fluorescein-labeled anti-tTG to QSY 7-labeled tTG. We expect that our FRET results could have applications in the development of a homogeneous FRET immunoassay for early diagnosis and followup of celiac patients. The FRET efficiency presented herein 
may be improved in several ways. First, we can think of using human recombinant tTG as the antigenic source. Second, monoclonal IgG or IgA anti-tTG will show better sensitivity. Third, we can change the donor. If we use longwavelength probes as the donor, we can substantially reduce the background emission or autofluorescence of the sample, which is the main limitation to highly sensitive fluorescence detection. Fourth, we can think of changing the acceptor. In this report, we used a nonfluorescent quencher QSY7 as the acceptor, so we measured the donor fluorescence only. When a fluorescent quencher was used, donor emission decreased and the acceptor emission increased upon FRET (D'Auria et al., 2012). Hence, by taking the ratio of the acceptor to the donor emission, a ratiometric sensor can be created. Quantum dots may be employed as the donor and/ or the acceptor of the FRET immunoassay because quantum dots have been estimated to be 20 times brighter and 100 times more stable than conventional fluorescent dyes. Further experimentation is required to test the above ideas.

Previous findings related to measurement of antibodies present in other autoimmune diseases (e.g., autoimmune diabetes) clearly showed the superiority both in sensitivity and specificity of fluid- with respect to solid-phase assays (Greenbaum et al., 1992). In fact, anti-tTG autoantibodies measurement by the fluid-phase radioimmunoassay (RIA) generally showed higher sensitivity than the solid-phase ELISA (Bonamico et al., 2001; Li et al., 2009). At disease diagnosis, when the anti-tTG autoantibodies are usually found at high titers, the performance of the salivary and of the three serum assays (RIA, ELISA and EMA) was quite similar, whereas during the gluten-free diet follow-up when the autoantibody titers tend to decrease progressively, salivary and serum RIA anti-tTG methods detect higher frequencies of autoantibody positive patients with respect to ELISA and EMA (Bonamico et al., 2008). In many fields of bioscience, fluorescence-based detection has successfully replaced radioactive isotope labeling. In recent years, there has been an increasing trend in using saliva as a diagnostic biofluid (Bonamico et al., 2008; Condò et al., 2013). If the sensitivity of anti-tTG autoantibodies assay can be improved using a homogeneous FRET immunoassay, it may be possible to use saliva instead of sera, which will enable to by-pass the unpleasant blood sample collection. This will be especially helpful in children for CD-screening purposes (Condò et al., 2013). Additionally, anti-endomysial and anti-tTG autoantibodies were detected in media following culture of oral biopsies from patients with untreated CD (Vetrano et al., 2007; Compilato et al., 2010). If we can develop a highly sensitive and specific biosensor for CD based on a homogeneous FRET immunoassay, definite diagnosis may be conducted using saliva and/or oral biopsies instead of intestinal biopsies. It is expected that the recent advances in microfluidics-based biochip technology will spur the realization of this.

\section{Acknowledgments}

This work was supported by a 2-year Research Grant of Pusan National University. JSK thanks Professor Mauro Rossi (Institute of Food Science, CNR, Avellino, Italy) for the gift of anti-tTG antibodies. JSK is also grateful to Professor Sabato D'Auria, Institute of Protein Biochemistry, CNR, Naples, Italy for providing instrumentation.

\section{Conflict of interest}

None.

\section{REFERENCES}

AGA Institute. AGA Institute medical position statement on the diagnosis and management of celiac disease. Gastroenterology. 2006. 131: 1977-1980.

Basso D, Guariso G, Fasolo M, Pittoni M, Schiavon S, Fogar P, Greco E, Navaglia F, Zambon CF, Plebani M. A new indirect chemiluminescent immunoassay to measure anti-tissue transglutaminase antibodies. J Pediatr Gastroenterol. 2006. 43: 613 -618 .

Basso D, Guariso G, Fogar P, Meneghel A, Zambon CF, Naviglia F, Greco E, Schiavon S, Rugge M, Plebani M. Antibodies against synthetic deamidated gliadin peptides for celiac disease diagnosis and follow-up in children. Clin Chem. 2009. 55: 150 -157 .

Bonamico M, Nenna R, Luparia RPL, Perricone C, Montuori M, Lucantoni F, Castronovo A, Mura S, Turchetti A, Strappini P, Tiberti C. Radioimmunological detection of anti-transglutaminase autoantibodies in human saliva: a useful test to 
monitor celiac disease follow-up. Aliment Pharmacol Ther. 2008. 28: 364-370.

Bonamico M, Tiberti C, Picarelli A, Mariani P, Rossi D, Cipolletta E, Greco M, Tola MD, Sabbatella L, Carabba B, Magliocca FM, Strisciuglio P, Di Mario U. Radioimmunoassay to detect antitransglutaminase autoantibodies is the most sensitive and specific screening method for celiac disease. Am J Gastroenterol. 2001. 96: 1536-1540.

Catassi C, Fasano A. Celiac disease. Curr Opin Gastroenterol. 2008. 24: 687-691.

Chorzelski TP, Beutner EH, Sulej J, Tchorzewska H, Jablonska S, Kumar V, Kapuscinska A. IgA anti-endomysium antibody. A new immunological marker of dermatitis herpetiformis and coeliac disease. Br J Dermatol. 1984. 111: 395-402.

Collin P, Maki M, Keyrilainen O, Hallstrom O, Reunala T, Pastenack

A. Selective IgA deficiency and coeliac disease. Scand J Gastroenterol. 1992. 27: 367-371.

Compilato D, Campisi G, Pastore L, Carroccio A. The production of the oral mucosa of antiendomysial and anti-tissuetransglutaminase antibodies in patients with celiac disease: a review. The Scientific World Journal. 2010. 10: 2385-2394.

Condò R, Costacurta M, Docimo R. The anti-transglutaminase auto-antibodies in children's saliva with a suspect coeliac disease: clinical study. Oral Implantol. 2013. 6: 48-54.

D'Auria S, Apicella E, Staiano M, Giovanni S, Riggiero G, Rossi M, Sarkar P, Luchowski R, Gryczinski I, Gryczinski Z. Engineering resonance energy transfer for advanced immunoassays: the case of celiac disease. Anal Biochem. 2012. 425: 13-17.

Dieterich W, Ehnis T, Bauer M, Donner P, Volta U, Riecken EO, Schuppan D. Indentification of tissue trnasglutaminase as the autoantigen of celiac disease. Nat Med. 1997. 3: 798-801.

Greenbaum CJ, Palmer JP, Kuglin B, Kolb H. Insulin autoantibodies measured by radioimmunoassay methodology are more related to insulin-dependent diabetes mellitus than those measured by enzyme-linked immunoassorbent assay: results of the fourth international workshop in the standardization of insulin autoantibody measurement. J Clin Endocrinol Metab. 1992. 74: 1040-1044.

Green PH, Jabri B. Coeliac disease. Lancet. 2003. 362: 383-391.

Hill ID. What are the sensitivity and specificity of serologic tests for celiac disease? Do sensitivity and specificity vary in different population?. Gastroenterology. 2005. 128: S25-32.

Hill ID, Dirks MH, Liptak GS, Colletti RB, Fasano A, Guandalini S, Hoffenberg EJ, Horvath K, Murray JA, Pivor M, Seidman EG. Guideline for the diagnosis and treatment of celiac disease in children: recommendations of the North American Society for Pediatric Gastroenterology, Hepatology and Nutrition. J Pediatr Gastroenterol Nutr. 2005. 40: 1-19.

Husby S, Koletzko S, Korponay-Szabo IR, Mearin ML, Phillips A, Shamir R, Troncone R, Giersiepen K, Branski D, Catassi C, Lelgeman M, Maki M, Ribes-Koninckx C, Ventura A, Zimmer KP. European Society for Pediatric Gastroenterology, Hepatology, and Nutrition guidelines for the diagnosis of coeliac disease. J Pediatr Gastroenterol Nutr. 2012. 54: 136-160.

Lakowicz JR. Principles of Fluorescence Spectroscopy. 2006. 443 $-451$.

Lakowicz JR, Laczko G, Cherek H, Gratton E, Limkeman M. Analysis of fluorescence decay kinetics from variablefrequency phase-shift and modulation data. Biophys J. 1984. 46: 463-477.

Leffler DA, Edwards George JB, Dennis M, Cook EF, Schuppan D, Kelly CP. A prospective comparative study of five measures of gluten-free diet adherence in adults with celiac disease. Aliment Pharmacol Ther. 2007. 26: 1227-1235.

Lenhardt A, Plebani A, Marchetti F, Gerarduzzi T, Not T, Meini A, Villanacci V, Martelossi S, Ventura A. Role of human-tissue transglutaminase IgG and anti-gliadin IgG antibodies in the diagnosis of coeliac disease in patients with selective immunoglobulin A deficiency. Dig Liver Dis. 2004. 36: 730-734.

Li M, Yu L, Tiberti C, Bonamico M, Taki I, Miao D, Murray JA, Rewers MJ, Hoffenberg EJ, Agardh D, Mueller P, Stern M, Bonifacio E, Liu E. A report on the international transglutaminase autoantibody workshop for celiac disease. Am J Gastroenterol. 2009. 104: 154-163.

Rittmeyer C, Rhoads JM. IgA deficiency causes false-negative endomysial antibody results in celiac disease. J Pediatr Gastroenterol Nutr. 1996. 23: 504-506.

Sollid LM. Molecular basis of celiac disease. Annu Rev Immunol. 2000. 18: 53-81.

Sollid LM, Jabri B. Triggers and drivers of autoimmunity: lessons from coeliac disease. Nat Rev Immunol. 2013. 13: 294-302.

Suh-Lailam BB, Davis KW, Tebo AE. Immunoassays for the detection of IgA antibodies to tissue transglutaminase: significance of multiples of the upper limit of normal and inter-assay correlations. Clin Chem Lab Med. 2016. 54: 257-264.

Vetrano S, Zampaletta U, Anania MC, Tola MD, Sabbatella L, Passarelli F, Maffia C, Sanjust MG, Lettieri F, De Pità O, Picarelli A. Detection of anti-endomysial and anti-tissue transglutaminase autoantibodies in media following culture of oral biopsies from patients with untreated coeliac disease. Dig Liver Dis. 2007. 39: 911-916. 